\title{
Twenty years of asymptotic correction for eigenvalue computation
}

\author{
Alan L. Andrew* \\ (Received 7 August 2000)
}

\begin{abstract}
Asymptotic correction, first studied systematically in the 1979 ANU thesis of John Paine, can significantly increase the accuracy and efficiency of finite difference and finite element methods for computing eigenvalues, especially higher eigenvalues, of differential operators. It has proved especially useful for the solution of inverse eigenvalue problems. This paper reviews the impact of this method, and also presents
\end{abstract}

* Mathematics Department, La Trobe University, Bundoora, VIC 3083, Australia. mailto: A.Andrew@latrobe.edu.au

${ }^{0}$ See http: //anziamj . austms .org. au/V42/CTAC99/Andr for this article and ancillary services, (C) Austral. Mathematical Soc. 2000. Published 27 Nov 2000. 
some new numerical results which support a recent conjecture of the author concerning the use of asymptotic correction with Numerov's method for problems with natural boundary conditions.

\section{Contents}

1 Introduction

C97

2 Recent developments

C101

3 Numerical results

C107

References

C111

\section{Introduction}

Since many classical methods for numerical solution of eigenvalue problems for differential operators involve approximation of the corresponding eigenfunctions by piecewise polynomials, they are inefficient for computation of higher eigenvalues. The 1979 ANU thesis of John Paine [24], written under the supervision of Frank de Hoog and Bob Anderssen, made three important contributions to the efficient and accurate computation of the higher eigenvalues of Sturm-Liouville problems. The first of these, which has not been 
published (though a brief summary of the main results is given in [27]), is a careful error analysis of some shooting methods based on the popular Prüfer transformation and its variants. The second $[25,28]$ describes how the Pruess method [33] can be implemented to obtain uniformly accurate eigenvalue estimates. However, it is the third contribution of [24], the development of a method which the author [2] has called the method of asymptotic correction, which has proved the most influential. The primary aim of this paper is to describe the impact of this third contribution of [24], and give an assessment of the current state of the art. In addition, some new numerical results are presented which support a recent conjecture of the author [11].

Only regular problems, with coefficients sufficiently smooth to allow the use of the Liouville transformation, were considered in [24]. For such problems, it was shown in [24] that preliminary transformation of the equation to the Liouville normal form

$$
-y^{\prime \prime}+q y=\lambda y
$$

improves the performance of all methods considered there for the computation of higher eigenvalues. Asymptotic correction, which has sometimes also been called "algebraic correction" [17] or "the AAdHP correction" [33], depends on the fact that the error in the computed eigenvalues is not particularly sensitive to changes in the potential $q$, and that, for many problems, this error can be computed in closed form when $q$ is constant. It was suggested in [24] that an approximation $\Lambda_{k}^{(n)}$ to an eigenvalue $\lambda_{k}$ be replaced by an approximation $\Lambda_{k}^{(n)}+\mu(n, k)$, where the "asymptotic correction", $\mu(n, k)$, 
which is independent of $q$, is chosen so that, in the special case of constant $q$, the corrected approximation, $\Lambda_{k}^{(n)}+\mu(n, k)$, equals $\lambda_{k}$ exactly. For example, when the classical second order centred finite difference method, with uniform mesh length $h=\pi / n$, is used to solve (1) with boundary conditions

$$
\begin{gathered}
y(0)=y(\pi)=0, \\
y^{\prime}(0)=y^{\prime}(\pi)=0, \\
y(0)=y^{\prime}(\pi)=0,
\end{gathered}
$$

or

$$
y^{\prime}(0)=y(\pi)=0,
$$

it is readily checked that the appropriate values of $\mu(n, k)$ are $\varepsilon_{1}(k, h), \varepsilon_{1}(k-$ $1, h), \varepsilon_{1}\left(k-\frac{1}{2}, h\right)$ and $\varepsilon_{1}\left(k-\frac{1}{2}, h\right)$ respectively, where, as in $[5,11]$,

$$
\varepsilon_{r}(k, h)=k^{2}-\frac{12 \sin ^{2}(k h / 2)}{h^{2}\left[3+(1-r) \sin ^{2}(k h / 2)\right]} .
$$

It was shown in [24], and by a slightly different proof in [29], that this correction reduces the error in the $k$ th eigenvalue of $(1),(2)$ obtained by the classical centred finite difference method with uniform mesh length $h$ from $O\left(k^{4} h^{2}\right)$ to $O\left(k h^{2}\right)$, provided $k h$ is "sufficiently small" and $q \in C^{2}$. Using some asymptotic formulae proved in [16], Anderssen and de Hoog [1] extended the results of [29] to problems with general separated boundary conditions, although, except in the cases mentioned above, a numerical calculation is 
required to determine the error for constant $q$. They also showed that, except in the case (2) which is discussed further in [2], the error in the corrected estimates is only $O\left(h^{2}\right)$, again provided $k h$ is "sufficiently small". For (1), (2) asymptotic correction has been extended to Numerov's method [12], for which the correction is $\mu(n, k)=\varepsilon_{2}(k, h)$. A slightly different method of proof [12] showed that, for all $k$, this correction reduces the error from the $O\left(k^{6} h^{4}\right)$ error of the uncorrected Numerov method [3] to $O\left(k^{4} h^{5} / \sin (k h)\right)$, provided $q \in C^{4}$. The method of proof in [12] has subsequently been used in the analysis of other methods [34] and can also be used to eliminate the restriction to "sufficiently small" $k h$ in $[24,29]$, provided the $O\left(k h^{2}\right)$ bound is replaced by $O\left(k^{2} h^{3} / \sin (k h)\right)$. Similar results have been obtained for finite element methods with linear hat coordinate functions $[4,13]$ and for periodic boundary conditions $[4,6]$. Finite element methods have proved especially accurate for midrange $k$, provided sufficiently accurate methods of numerical quadrature are used to evaluate the matrix elements. This proviso is required because quadrature errors, which would be swamped by other errors in the uncorrected estimates, can dominate the much smaller errors in the corrected estimates $[9,13]$.

A survey of results on asymptotic correction up until 1992 is given in [7], which also describes some open questions. Section 2 below complements [7] by assessing subsequent work, especially progress that has been made on the questions posed in [7]. Not surprisingly, asymptotic correction has proved most successful when the derivatives of $q$ (including the higher derivatives) are small, and many of the results discussed here emphasize this fact. 


\section{Recent developments}

The first question posed in [7] was "For which other problems and other methods will asymptotic correction produce significant improvement?". As described below, several other applications of asymptotic correction have subsequently been suggested and their usefulness supported by numerical results [20], but a complete theory has been given for only a few of these. The proof given in [6] for solution of Sturm-Liouville problems with periodic boundary conditions using a classical finite difference scheme has been extended [15] to a refined difference scheme suggested in [35]. In the important special case $q^{\prime}(0)=q^{\prime}(\pi)$, the two schemes coincide and a second scheme suggested in [35] reduces to a multiplicative asymptotic correction similar to that used in [23]. As emphasized in [15], where the relationship between the methods of [6] and [35] is clarified, the proof in [6] does not depend on any assumption about the value of $q^{\prime}(0)-q^{\prime}(\pi)$.

Another important development has been an extension [11] of the method of [12] to problems with boundary conditions (3), (4), and (5). As in [11], we denote the $k$ th eigenvalue of (1) with boundary conditions (2), (3), (4), and (5) by $\lambda_{k}^{(0)}, \lambda_{k}^{(1)}, \lambda_{k}^{(2)}$, and $\lambda_{k}^{(3)}$, respectively $\left(\lambda_{1}^{(i)}<\lambda_{2}^{(i)}<\cdots\right)$ and the uncorrected and corrected Numerov approximations of $\lambda_{k}^{(i)}$ with $n$ equal subintervals by $\Lambda_{k}^{(i, n)}$ and $\tilde{\Lambda}_{k}^{(i, n)}$, respectively. In [11] the author derived the appropriate fourth order approximation of the boundary conditions (3), (4), and $(5)$ and showed that the appropriate form of the correction for these three boundary conditions was $\epsilon_{2}(k-1, h), \epsilon_{2}\left(k-\frac{1}{2}, h\right)$ and $\epsilon_{2}\left(k-\frac{1}{2}, h\right)$, respectively. 
He also tested the method numerically and, on the basis of these numerical results and by analogy with results proved in [4, 12], conjectured that, for $i=1,2,3$ and all $k, n$ and all $q \in C^{4}[0, \pi]$,

$$
\lambda_{k}^{(i)}-\tilde{\Lambda}_{k}^{(i, n)}=O\left(k^{4} h^{5} / \sin ((k-1 / 2) h)\right) .
$$

Note that, for $i=0,(7)$ is an immediate consequence of a result proved in [12]. Some further numerical tests of this conjecture are reported here in the next section.

While most work on asymptotic correction has dealt with Sturm-Liouville problems, there is no reason in principle why it should not be successful for other differential operators, and early results [2] showed it could dramatically improve the accuracy of computed eigenvalues (including low eigenvalues) of partial differential operators. The single numerical example considered in [2] involved the equation

$$
-\nabla^{2} u+q u=\lambda u
$$

with $u$ required to vanish on the boundary of a rectangle. For this special problem, closed form solutions are available for constant $q$. For example, when $u$ is required to vanish at the boundaries of the square $[0, \pi] \times[0, \pi]$, the eigenvalues of (8) with $q=0$ are $k_{1}^{2}+k_{2}^{2}$, where $k_{1}, k_{2}=1,2,3 \ldots$, and, when the standard 5-point second order difference scheme is used with constant mesh length $h$ along both axes, the appropriate correction for the corresponding eigenvalues of (8) for general $q$ is then $\varepsilon_{1}\left(k_{1}, h\right)+\varepsilon_{1}\left(k_{2}, h\right)$. There is still no adequate theory for this problem, but it has since been considered in [17] where most of the discussion concerns ordering of the eigenvalues. Much is 
made in [17] of the fact that the first $N_{1}$ values of $n_{1}$ and the first $N_{2}$ values of $n_{2}$ do not determine the first $N_{1} N_{2}$ values of $n_{1}^{2}+n_{2}^{2}$, but since one is unlikely to attempt to compute $m$ eigenvalues of (8) using a matrix of dimension less than $2 m$ this is unlikely to prove a serious impediment to the use of asymptotic correction. Nevertheless, ordering of eigenvalues needs more care than with (1). For nonconstant $q$, the repeated eigenvalues in the sequence, $2\left(=1^{2}+1^{2}\right), 5\left(=1^{2}+2^{2}\right), 5\left(=2^{2}+1^{2}\right), 8,10,10,13,13,17, \ldots$, of eigenvalues for $q=0$ are usually replaced by sets of close eigenvalues, and it may not be clear which one corresponds to which eigenvalue of the $q=$ constant case. As is the case for all close eigenvalues of the problems considered in $[4,6]$, the early repeated eigenvalues cause no trouble, since the appropriate correction is the same for each member of the set. For instance, for the second and third it is $\varepsilon_{1}(1, h)+\varepsilon_{1}(2, h)$. However, unless the eigenfunctions provide clear evidence, it will be difficult to tell whether the correction for the $31^{\text {st }}$ eigenvalue should be $\varepsilon_{1}(5, h)+\varepsilon_{1}(5, h)$ or $\varepsilon_{1}(1, h)+\varepsilon_{1}(7, h)$ since $5^{2}+5^{2}=1^{2}+7^{2}$. The author suspects that, for the computation of a moderate number of eigenvalues when the derivatives of $q$ are small, the problem of ordering is not likely to be too serious. When $q$ itself is more variable, the perturbation in $\lambda_{k}$ it produces is more likely to vary substantially with $k$. In this case, a more serious problem than the repeated eigenvalues is probably the fact that the distinct eigenvalues of this problem for (8) are more likely to be clustered than the distinct eigenvalues of regular Sturm-Liouville problems. With sufficiently nasty $q$, even moderately close eigenvalues could cause difficulties, and, for example, it could even be difficult to tell whether the correction for the fourth eigenvalue should be $\varepsilon_{1}(2, h)+\varepsilon_{1}(2, h)$ or $\varepsilon_{1}(3, h)+\varepsilon_{1}(1, h)$. 
This is another reason why asymptotic correction is most useful when the derivatives of $q$ are small. Probably a more significant limitation is the fact that simple boundaries like squares are not the norm in real applications, and computing the solution with more general boundary conditions is often not much easier with constant $q$ than with general $q$. It is not surprising that asymptotic correction, like virtually all other methods, encounters greater difficulty with partial differential equations. This is not a reason to abandon the strategy but rather to refine it, perhaps using the suggestion of [2] that results for simpler boundary conditions could be used to correct results for more difficult boundary conditions.

Most work so far has been on individual cases, and the fact that the errors in the corrected estimates do seem to exhibit somewhat different behaviour in different cases suggests that consideration of individual cases may produce sharper results than could be obtained by a general theory. Nevertheless, a general theory of asymptotic correction could perhaps provide valuable pointers for future research as well as better overall understanding. Perhaps the clue to a general theory is provided by the fact that the addition of $q$ represents a bounded symmetric perturbation to an unbounded self-adjoint operator with compact inverse. Although the individual matrix approximations are bounded, their norms increase without bound as the mesh is refined, but the norm of the perturbations to these matrices produced by $q$ is uniformly bounded. Perhaps recent results on continuous dependence of eigenvalues on the coefficients [21] could also provide a clue, though it is finite perturbations that are involved here and, even in the finite dimensional case, the relationship between finite and continuous perturbations is 
not simple [10].

The last question asked in [7] was "How may asymptotic correction most effectively be used in the numerical solution of inverse eigenvalue problems?". Matrix inverse eigenvalue problems [14] are generally easier to solve numerically than corresponding problems for differential equations. Consequently, several papers on matrix inverse eigenvalue problems have cited possible application to the inverse Sturm-Liouville problem as motivation. However, an appropriate implementation of asymptotic correction is required for the viability of most such methods for the solution of inverse Sturm-Liouville problems [22, 26]. Only $q$ satisfying $q(\pi-x)=q(x)$ was considered in [26], and a simple centred finite difference approach was used there. A standard algorithm for the matrix inverse eigenvalue problem was used to obtain the tridiagonal matrix, and information was extracted from the computed off-diagonal elements using a discrete Liouville transformation. A simpler method of extracting this information has since been suggested in [17, 18]; these also give a fuller theoretical treatment than that given in [26]. The use of asymptotic correction in solving inverse eigenvalue problems is discussed further in [8], where other important methods are also considered. Other methods using asymptotic correction [19, 22, 30, 31] construct only the diagonal elements, using the fact that the off-diagonal elements are already known, so that $m$ eigenvalues suffice for a $2 m \times 2 m$ matrix. An advantage of this approach is that it uses only those eigenvalues for which asymptotic correction is most successful. As noted in [17], where several difficulties of the corresponding problem for partial differential equations are discussed, this advantage is likely to be especially important for partial differential 
equations. Fabiano, Knobel and Lowe [19] gave algorithms using asymptotic correction for the inverse Sturm-Liouville eigenvalue problem not only in the case $q(\pi-x)=q(x)$, but also for general $q$. In the latter case, both eigenvalues and terminal velocities are used as data. They showed that, for all $m$ (the number of eigenvalues used), there exists a constant $C(m)$ such that their algorithms (which are based on the modified Newton's method) always converge if there is a constant $q_{0}$ such that the $L_{2}$ norm of $\left(q-q_{0}\right)$ is less than $C(m)$. Numerical results indicate that $C(m)$ need not be particularly small. Because alternative methods are less well developed for the inverse problem than for the forward problem, and because the different asymptotic behaviour of the eigenvalues of the differential equation and its discretization has particularly serious consequences for solution of the inverse problem $[8,17,22,26,30]$, it is likely that the solution of inverse eigenvalue problems will prove to be one of the most important applications of asymptotic correction.

There has been less progress on the other questions posed in [7]. The question concerning sharper results on the dependence on $k$ and $h$ of the error in the corrected results, and the application of this to extrapolation, is discussed briefly in the next section. Regarding the question, "Under what circumstances can we be sure that asymptotic correction produces an improvement for all $k$ ?", it is now known [11] that, when $n$ is small, asymptotic correction can sometimes produce a deterioration for some $\lambda_{k}$ with $k>1$. Only cases with $k=1$ were reported in [7]. With boundary conditions (3) and periodic boundary conditions, asymptotic correction produces no change (but also requires no work) for the fundamental. With this minor exception, 
the only cases known to the author, in which asymptotic correction fails to produce improvement for all eigenvalues, involve a coarser mesh than is likely to be be used in practical computation. Moreover, even with small $n$, asymptotic correction has still produced improvement in the overwhelming majority of cases. So far there is no evidence to challenge the conjecture of [7] regarding the success of asymptotic correction for all "sufficiently large" $n$. A mystery not mentioned in [7] is the table on p 123 of [24], which claims to give results for the corrected and uncorrected solutions of (1), (4) using the classical second order centred finite difference method, and which suggests that there are other cases in which the correction produces a deterioration. Since the errors shown in that table differ by an order of magnitude from those given for the same calculation in Table 5 of [29], the author has repeated the calculations. Apart from minor differences in the least significant figure, his results agreed with those given in [29], which show that asymptotic correction increases accuracy for all $k$.

\section{$3 \quad$ Numerical results}

Numerov's method approximates (1) by the three term recurrence relation

$$
\begin{array}{r}
-\left[12-h^{2} q\left(x_{i-1}\right)\right] y_{i-1}+\left[24+10 h^{2} q\left(x_{i}\right)\right] y_{i}-\left[12-h^{2} q\left(x_{i+1}\right)\right] y_{i+1} \\
=h^{2} \Lambda\left[y_{i-1}+10 y_{i}+y_{i+1}\right] .
\end{array}
$$


All numerical results in [11] were for problems with boundary conditions (3) and with $q^{\prime}$ vanishing at both boundaries. The boundary conditions simplify slightly when $q^{\prime}$ vanishes at a boundary, and the corrected Numerov method of [11] has not previously been tested on examples with boundary conditions (4) or (5). To complement the numerical tests of [11], we give here some numerical tests of the corrected Numerov method with boundary conditions (4) and $q^{\prime}(\pi) \neq 0$. As shown in [11], a suitable fourth order approximation of the boundary conditions (4) is given by $y_{0}=0$ and

$$
\begin{array}{r}
-\left[24-h^{2}\left(q\left(x_{n-1}\right)+q\left(x_{n+1}\right)\right)\right] y_{n-1}+\left[24+10 h^{2} q\left(x_{n}\right)\right. \\
\left.-h^{3} q^{\prime}\left(x_{n}\right)\left(4-h^{2} q\left(x_{n+1}\right) / 3\right)\right] y_{n}=h^{2} \Lambda\left[2 y_{n-1}+\left(10+h^{3} q^{\prime}\left(x_{n}\right) / 3\right) y_{n}\right] .
\end{array}
$$

Table 1 shows some results for $q(x)=e^{x}$. This example was also used in $[4,24,29]$. The results in the last four columns are clearly consistent with (7). To avoid clutter in the headings, the superscript " 2 " is omitted, with $\lambda_{k}^{(2)}, \Lambda_{k}^{(2,40)}$ etc written as $\lambda_{k}, \Lambda_{k}^{(40)}$ etc. The "exact" $\lambda_{k}$ used in this table was $C_{k}(240,180)$, using the extrapolation formula

$$
C_{k}(n, m)=\frac{n^{5} \sin \left(\left(k-\frac{1}{2}\right) \pi / n\right) \tilde{\Lambda}_{k}^{(2, n)}-m^{5} \sin \left(\left(k-\frac{1}{2}\right) \pi / m\right) \tilde{\Lambda}_{k}^{(2, m)}}{n^{5} \sin \left(\left(k-\frac{1}{2}\right) \pi / n\right)-m^{5} \sin \left(\left(k-\frac{1}{2}\right) \pi / m\right)}
$$

suggested by $(7)$. Comparison with $C_{k}(200,150)$ and $C_{k}(160,120)$ suggests that all figures shown are correct.

The results will not be changed (apart from a small amount due to roundoff) when $q$ is increased by a constant, but we may expect an increase in error 
TABLE 1: Errors in computed solutions of (1), (4) with $q(x)=e^{x}$

\begin{tabular}{|c|c|cc|cccc|}
\hline & & & & \multicolumn{4}{|c|}{$10\left(\lambda_{k}-\tilde{\Lambda}_{k}^{(n)}\right) \sin \left(\left(k-\frac{1}{2}\right) h\right) /\left(k^{4} h^{5}\right)$} \\
\cline { 5 - 8 }$k$ & $\lambda_{k}$ & $\lambda_{k}-\Lambda_{k}^{(40)}$ & $\lambda_{k}-\tilde{\Lambda}_{k}^{(40)}$ & $n=10$ & $n=20$ & $n=40$ & $n=80$ \\
\hline 1 & 4.89571 & $2.52 \mathrm{E}-6$ & $2.52 \mathrm{E}-6$ & 0.333 & 0.331 & 0.331 & 0.331 \\
2 & 9.99955 & $3.04 \mathrm{E}-5$ & $2.87 \mathrm{E}-5$ & 0.706 & 0.703 & 0.705 & 0.705 \\
3 & 15.4685 & $8.41 \mathrm{E}-5$ & $4.54 \mathrm{E}-5$ & 0.368 & 0.362 & 0.366 & 0.367 \\
4 & 21.0371 & $3.86 \mathrm{E}-4$ & $9.35 \mathrm{E}-5$ & 0.275 & 0.319 & 0.332 & 0.335 \\
5 & 28.1893 & $1.80 \mathrm{E}-3$ & $4.76 \mathrm{E}-4$ & 0.648 & 0.835 & 0.882 & 0.894 \\
6 & 37.7907 & $5.40 \mathrm{E}-3$ & $9.76 \mathrm{E}-4$ & 0.661 & 0.972 & 1.055 & 1.075 \\
7 & 49.6137 & $1.36 \mathrm{E}-2$ & $1.53 \mathrm{E}-3$ & 0.529 & 0.926 & 1.040 & 1.069 \\
8 & 63.5205 & $3.07 \mathrm{E}-2$ & $2.15 \mathrm{E}-3$ & 0.397 & 0.832 & 0.977 & 1.013 \\
9 & 79.4646 & $6.37 \mathrm{E}-2$ & $2.86 \mathrm{E}-3$ & 0.223 & 0.731 & 0.902 & 0.947 \\
10 & 97.4279 & $1.23 \mathrm{E}-1$ & $3.65 \mathrm{E}-3$ & -0.318 & 0.632 & 0.829 & 0.881 \\
11 & 117.402 & $2.22 \mathrm{E}-1$ & $4.54 \mathrm{E}-3$ & & 0.542 & 0.761 & 0.821 \\
12 & 139.384 & $3.83 \mathrm{E}-1$ & $5.51 \mathrm{E}-3$ & & 0.459 & 0.698 & 0.765 \\
13 & 163.370 & $6.33 \mathrm{E}-1$ & $6.58 \mathrm{E}-3$ & & 0.386 & 0.641 & 0.715 \\
14 & 189.359 & $1.01 \mathrm{E} \mathrm{0}$ & $7.74 \mathrm{E}-3$ & & 0.323 & 0.589 & 0.669 \\
15 & 217.351 & $1.55 \mathrm{E} \mathrm{0}$ & $9.01 \mathrm{E}-3$ & & 0.271 & 0.541 & 0.628 \\
16 & 247.344 & $2.32 \mathrm{E} \mathrm{0}$ & $1.04 \mathrm{E}-2$ & & 0.231 & 0.497 & 0.591 \\
17 & 279.338 & $3.40 \mathrm{E} \mathrm{0}$ & $1.18 \mathrm{E}-2$ & & 0.207 & 0.457 & 0.557 \\
18 & 313.334 & $4.85 \mathrm{E} \mathrm{0}$ & $1.34 \mathrm{E}-2$ & & 0.204 & 0.420 & 0.526 \\
19 & 349.330 & $6.81 \mathrm{E} \mathrm{0}$ & $1.51 \mathrm{E}-2$ & & 0.161 & 0.385 & 0.497 \\
20 & 387.326 & $9.38 \mathrm{E} \mathrm{0}$ & $1.69 \mathrm{E}-2$ & & -0.368 & 0.353 & 0.471 \\
39 & 1489.30 & $5.15 \mathrm{E} \mathrm{2}$ & $8.20 \mathrm{E}-1$ & & & 0.139 & 0.192 \\
40 & 1567.30 & $5.81 \mathrm{E} \mathrm{2}$ & $-7.47 \mathrm{E} 0$ & & & -0.383 & 0.183 \\
\hline
\end{tabular}


when the derivatives of $q$ are increased in magnitude. In order to test the size of this increase, most of the above calculations were repeated for $q(x)=4 e^{x}$. For $q(x)=e^{x}$, the value of the scaled error

$$
\theta(k, h)=\left(\lambda_{k}^{(2)}-\tilde{\Lambda}_{k}^{(2, n)}\right) \sin ((k-1 / 2) h) /\left(k^{4} h^{5}\right)
$$

(which is multiplied by 10 in Table 1 for ease of tabulation) varied in magnitude from a maximum of just over 0.1 at $k=6$ and 7 to a minimum of about 0.01 at $k=n-1$ (with a sign change only for $k=n$ ). For $q(x)=4 e^{x}$, $\theta(k, h)$ varied from a maximum of just over 1.0 for $k=4$ and 5 , to a minimum of about 0.07 at $k=n-1$, with the sign change at $k=n-1$ when $n \geq 20$. These results, like those of [11], suggest that the dependence of the error on the derivatives of $q$ is mildly superlinear. They also suggest that the error estimate (7) is sharper for (1), (4) than those proved in [4, 12, 13, 29]. The regularity of these results allows extrapolation using (11) to be useful over a wider range than is the case with the corrected second order results, although, as with the corrected second order methods [5], the best results are likely when the ratio of the values of $n$ used for extrapolation is less than two. Extrapolation has already been shown to be very effective with the corrected Numerov method for (2) as, although the results proved in [12] are not sharp, the stronger results conjectured there, which appear to be sharp [2], produce the same extrapolation formula [7]. The conjecture of [32] that further extrapolation of the corrected second order results could produce the same benefits as Numerov's method appears to have been made on the assumption that the appropriate extrapolation formula was the same for corrected as for uncorrected estimates. Our results indicate that much better 
accuracy can be obtained with formulae such as (11).

\section{References}

[1] R. S. Anderssen and F. R. de Hoog. On the correction of finite difference eigenvalue approximations for Sturm-Liouville problems with general boundary conditions. BIT, 24:401-412, 1984. C99

[2] A. L. Andrew. Asymptotic correction of finite difference eigenvalues. In J. Noye and R. May, editors, Computational Techniques and Applications: CTAC85, pages 333-341, Amsterdam, 1986. North-Holland. C98, C100, C102, C102, C104, C110

[3] A. L. Andrew. The accuracy of Numerov's method for eigenvalues. BIT, 26:251-253, 1986. C100

[4] A. L. Andrew. Correction of finite element eigenvalues for problems with natural or periodic boundary conditions. BIT, 28:254-269, 1988. C100, C100, C102, C103, C108, C110

[5] A. L. Andrew. Efficient computation of higher Sturm-Liouville eigenvalues. Internat. Schriftenreihe Numer. Math. Birkhäuser, 86:1-9, 1988. C99, C110 
[6] A. L. Andrew. Correction of finite difference eigenvalues of periodic Sturm-Liouville problems. J. Austral. Math. Soc. Ser. B, 30:460-469, 1989. C100, C101, C101, C101, C103

[7] A. L. Andrew. Asymptotic correction of computed eigenvalues of differential equations. Ann. Numer. Math., 1:41-51, 1994. C100, C100, C100, C101, C105, C106, C106, C107, C107, C110

[8] A. L. Andrew. Some recent developments in inverse eigenvalue problems. In D. Stewart, D. Singleton, and H. Gardner, editors, Computational Techniques and Applications: CTAC93, pages 94-102, Singapore, 1994. World Scientific. C105, C106

[9] A. L. Andrew. Quadrature errors in finite element eigenvalue computations. ANZIAM J., 42:136-140, 2000. C100

[10] A. L. Andrew. Finite and continuous perturbations of matrix eigenvalues. Appl. Math. Lett., 11(no. 1):47-51, 1998. C105

[11] A. L. Andrew. Asymptotic correction of Numerov's eigenvalue estimates with natural boundary conditions. J. Comput. Appl. Math., 125 to appear. C98, C99, C101, C101, C101, C106, C108, C108, C108, C108, C110

[12] A. L. Andrew and J. W. Paine. Correction of Numerov's eigenvalue estimates. Numer. Math., 47:289-300, 1985. C100, C100, C100, C101, C102, C102, C110, C110 
[13] A. L. Andrew and J. W. Paine. Correction of finite element estimates for Sturm-Liouville eigenvalues. Numer. Math., 50:205-215, 1986. C100, C100, C110

[14] M. T. Chu. Inverse eigenvalue problems. SIAM Rev., 40:1-39, 1998. $\mathrm{C} 105$

[15] D. J. Condon. Corrected finite difference eigenvalues of periodic Sturm-Liouville problems. Appl. Numer. Math., 30:393-401, 1999. C101, C101

[16] F. R. de Hoog and R. S. Anderssen. Asymptotic formulas for discrete eigenvalue problems in Liouville form. Math. Mod. Meth. Appl. Sci., to appear. C99

[17] C. R. Dun. Algebraic correction methods for two-dimensional eigenvalue problems. PhD thesis, Australian National University, Canberra, Australia, 1995. C98, C102, C103, C105, C105, C106

[18] C. R. Dun and R. S. Anderssen. A modification of Paine's algebraic correction method for inverse Sturm-Liouville problems. Preprint, 1995. C105

[19] R. H. Fabiano, R. Knobel and B. D. Lowe. A finite difference algorithm for an inverse Sturm-Liouville problem. IMA J. Numer. Anal., 15:75-88, 1995. C105, C106 
[20] P. Ghelardoni. Approximations of Sturm-Liouville eigenvalues using boundary value methods. Appl. Numer. Math., 23:311-325, 1997. C101

[21] Q. Kong, H. Wu, and A. Zettl. Dependence of the $n$th Sturm-Liouville eigenvalue on the problem. J. Differential Equations, 156:328-354, 1999. C104

[22] J. T. Marti. Small potential corrections for the discrete eigenvalues of the Sturm-Liouville problem. Numer. Math., 57:51-62, 1990. C105, C105, C106

[23] F. Natterer. A Lanczos type algorithm for inverse Sturm-Liouville problems. Proc. Centre Math. Appl. Austral. Nat. Univ., 31:82-88, 1992. C101

[24] J. W. Paine. Numerical approximation of Sturm-Liouville eigenvalues. PhD thesis, Australian National University, Canberra, Australia, 1979. C97, C98, C98, C98, C98, C98, C99, C100, C107, C108

[25] J. W. Paine. Correction of Sturm-Liouville eigenvalue estimates. Math. Comp., 39:415-420, 1982. C98

[26] J. W. Paine. A numerical method for the inverse Sturm-Liouville problem. SIAM J. Sci. Stat. Comput., 5:149-156, 1984. C105, C105, C105, C106 
[27] J. W. Paine and R. S. Anderssen. Uniformly valid approximation of eigenvalues of Sturm-Liouville problems in geophysics. Geophys.

J. Roy. Astronom. Soc., 63:441-465, 1980. C98

[28] J. W. Paine and F. R. de Hoog . Uniform estimation of the eigenvalues of Sturm-Liouville problems. J. Austral. Math. Soc., Ser. B, 21:365-383, 1980. C98

[29] J. W. Paine, F. R. de Hoog, and R. S. Anderssen. On the correction of finite difference eigenvalue approximations for Sturm-Liouville problems. Computing, 26:123-139, 1981. C99, C99, C100, C107, C107, C108, C110

[30] M. H. Pirovino. Das Sturm-Liouville-Problem als diskretes und inverses Eigenwertproblem und sein numerische Behandlung durch finite Differenzen. Dissertation, ETH Zürich, 1992. C105, C106

[31] M. H. Pirovino. The inverse Sturm-Liouville problem and finite differences. Sem. Angew. Math. ETH Zürich, Res. Rep., 93-04, 1993. C105

[32] M. B. Porter and E. L. Reiss. A numerical method for ocean-acoustic normal modes. J. Acoust. Soc. Amer., 76:244-252, 1984. C110

[33] J. D. Pryce. Numerical solution of Sturm-Liouville problems. Oxford Univ. Press, London, 1993. C98, C98 
[34] G. Vanden Berghe and H. De Meyer. Accurate computation of higher Sturm-Liouville eigenvalues. Numer. Math., 59:243-254, 1991. C100

[35] G. Vanden Berghe, M. Van Daele, and H. De Meyer. A modified difference scheme for periodic and semiperiodic Sturm-Liouville problems. Appl. Numer. Math., 18:69-78, 1995. C101, C101, C101 